Table 2 Side effect of iron chelating agents and drugs used in the treatment of cortical venous thrombosis ${ }^{6,10}$

\begin{tabular}{|l|l|}
\hline Drug & Side effects \\
\hline Decitabine & $\begin{array}{l}\text { Neutropenia, thrombocytopenia, anemia, } \\
\text { fatigue, pyrexia, nausea, cough, petechiae, } \\
\text { constipation, diarrhea, and hyperglycemia }\end{array}$ \\
\hline Hydroxycarbamide & $\begin{array}{l}\text { Increased risk of infection, breathlessness, } \\
\text { bruising, bleeding gums or nosebleeds, } \\
\text { fever, fatigue }\end{array}$ \\
\hline Desferrioxime & $\begin{array}{l}\text { Ocular and retinal toxicity, growth } \\
\text { impairment, ototoxicity, thrombocyto- } \\
\text { penia, abdominal pain, diarrhea, nausea, } \\
\text { vomiting and hypotension, increased risk } \\
\text { of infection }\end{array}$ \\
\hline
\end{tabular}

\section{Conflict of Interest}

None declared.

\section{References}

1 Kallenbach T. Anaesthesia for a patient with beta thalassaemia major. South African J Anaesth Analg [Internet] 2015;21(5):21-24

2 Staikou C, Stavroulakis E, Karmaniolou I. A narrative review of peri-operative management of patients with thalassaemia. Anaesthesia 2014;69(5):494-510
3 Saposnik G, Barinagarrementeria F, Brown RD Jr, et al; American Heart Association Stroke Council and the Council on Epidemiology and Prevention. Diagnosis and management of cerebral venous thrombosis: a statement for healthcare professionals from the American Heart Association/American Stroke Association. Stroke 2011;42(4):1158-1192

4 Yasmin A, O’Keefe YA, Kranz PG, Dombrowski KE, Kolls BJ, James ML. Cerebral venous thrombosis: reviewing pathophysiology, diagnosis, and treatment strategies. J Neuroanaesth Crit Care 2019;06:140-144

5 Keller E, Pangalu A, Fandino J, Könü D, Yonekawa Y. Decompressive craniectomy in severe cerebral venous and dural sinus thrombosis. Acta Neurochir Suppl (Wien) 2005;94(94):177-183

6 Kalantri SA, Ray R, Chattopadhyay A, Bhattacharjee S, Biswas A, Bhattacharyya M. Efficacy of decitabine as hemoglobin $\mathrm{F}$ inducer in $\mathrm{HbE} / \mathrm{\beta}$-thalassemia. Ann Hematol 2018;97(9):1689-1694

7 Succar J, Musallam KM, Taher AT. Thalassemia and venous thromboembolism. Mediterr J Hematol Infect Dis 2011;3(1):e2011025

8 Bharti N, Devrajan J. Difficult airway management in a case of thalassaemia major. Indian J Anaesth 2008;52(1):87-89

9 Eldor A, Rachmilewitz EA. The hypercoagulable state in thalassemia. Blood 2002;99(1):36-43

10 Mobarra N, Shanaki M, Ehteram H, et al. A review on iron chelators in treatment of iron overload syndromes. Int J Hematol Oncol Stem Cell Res 2016;10(4):239-247

\title{
Left Frontal Lobe Tumor-Induced Intraoperative Premature Ventricular Beats
}

\author{
Parmod K. Bithal ${ }^{1, \odot}$ Ravees Jan ${ }^{1} \quad$ Bharani Kumar ${ }^{1} \quad$ Insha ur Rahman ${ }^{1}$ \\ ${ }^{1}$ Department of Anesthesia and Perioperative Medicine, King Fahad \\ Medical City Riyadh, Riyadh, Saudi Arabia \\ J Neuroanaesthesiol Crit Care 2021;8:220-222. \\ Address for correspondence Ravees Jan, MBBS, MD, EDAIC, \\ CHRM, Department of Anesthesia and Perioperative Medicine, \\ King Fahad Medical City Riyadh 11525, Saudi Arabia \\ (e-mail: janravees@gmail.com).
}

Abstract

published online

October 24, 2021
In the absence of cardiac pathology, premature ventricular contractions (PVCs) in neurosurgical patients frequently accompany subarachnoid hemorrhage, intracerebral hemorrhage, traumatic brain injury, or raised intracranial pressure. PVCs detected during preanesthesia assessment prompts detailed cardiac evaluation. Our 57-year-old 


\section{Keywords}

- left frontal lobe tumor

- premature ventricular contractions

- lignocaine

- autonomic nervous system imbalance patient, a case of left frontal meningioma, with controlled hypertension, diabetes and hypothyroidism, had normal preoperative ECG and potassium. However, immediately on anesthesia induction, she developed multiple refractory to treatment PVCs but with normal blood pressure. Anesthesia, which was maintained with sevoflurane and fentanyl, was deepened to exclude light anesthesia as the cause, without useful outcome. Two lignocaine boluses (100 $\mathrm{mg}$ each), followed by its infusion, also proved ineffective. Her blood gases and potassium, checked twice, were normal. Throughout, her hemodynamics remained stable. As soon as tumor was removed, the PVCs disappeared not to return. Her postoperative recovery was uneventful with normal ECG.

\section{Introduction}

ECG changes in a neurosurgical patient in the absence of acute coronary event are known to occur following aneurysmal subarachnoid hemorrhage (aSAH), intracerebral hemorrhagic stroke, and traumatic brain injury (TBI). Whereas ECG changes are transient and subside without any treatment in the aSAH, in TBI, these changes revert to normal following treatment of underlying pathology. ${ }^{1}$ Another source of arrhythmias in neurosurgical patients is secondary to disruption of autonomic nervous system (ANS) activity from neoplasms involving vital brain areas. ${ }^{2}$ Primary brain areas involved in autonomic control of the brain-heart association are medial prefrontal cortex (mPFC), insular cortex (IC), and cerebellum. The right IC regulates sympathetic tone and left IC regulates parasympathetic tone. The MPFC lies at the crux of cortical regulation of visceral functions. ${ }^{3}$ Thus, a frontal lobe (FL) lesion might disrupt ANS activity, thereby creating an imbalance between parasympathetic and sympathetic activity, leading to arrhythmias. None of the reports so far has described arrhythmias resulting from a FL lesion under anesthesia, which disappeared with the excision of lesion.

We present a case report of an otherwise healthy female with left FL meningioma who developed multiple, unifocal, treatment refractory, premature ventricular contractions (PVCs) intraoperatively, which subsided, coinciding with the tumor excision.

\section{Case Report}

A 59-year-old, $71 \mathrm{~kg}$, American Society of Anesthesiologists (ASA) II female, diagnosed with left FL meningioma, was scheduled for craniotomy and tumor excision. She was a known case of hypertension, diabetes, and hypothyroidism on medications. After admission, she was started on dexamethasone. Her complete blood count, serum potassium (4.1 mEq/L) blood glucose, and ECG were normal. Her thyroid-stimulating hormone (TSH) level was a tad on the higher side of $6.098 \mathrm{mIU} / \mathrm{L}$ (normal 0.35-4.94) with $50 \mathrm{mcg} /$ day levothyroxine since 1 year, and normal T3 and T4 levels. Patient had good effort tolerance. Anesthesia was induced with fentanyl ( $2 \mathrm{mcg} / \mathrm{kg}$ ) and propofol $(2.5 \mathrm{mg} / \mathrm{kg})$, and trachea intubated following rocuronium ( $1 \mathrm{mg} / \mathrm{kg})$. At this stage, she developed multiple, monophasic PVCs ( $\geq 10$ per min); however, her blood pressure (BP) was normal. Besides routine monitoring, invasive radial artery pressure was also instituted. Mechanical ventilation was adjusted to maintain end tidal carbon dioxide around $32 \mathrm{~mm} \mathrm{Hg}$. Anesthesia was maintained with 50\% oxygen in air, sevoflurane, and infusions of fentanyl $(1 \mathrm{mcg} / \mathrm{kg} / \mathrm{h})$ and rocuronium. PVCs remained unabated despite two boluses of $100 \mathrm{mg}$ lignocaine each at 10 minutes apart. She received dexamethasone $8 \mathrm{mg}$ at induction and $0.5 \mathrm{gm} / \mathrm{kg}$ mannitol during craniotomy. To exclude light plane of anesthesia as the cause of PVCs, additional fentanyl $50 \mathrm{mcg}$ was given, and sevoflurane increased to 1 minimum alveolar concentration (MAC) from 0.8 MAC, but PVCs continued at the same frequency. Arterial blood gas analysis (ABG) revealed normal potassium $(3.9 \mathrm{mEq} / \mathrm{L})$ and $\mathrm{PaCO}_{2} 36 \mathrm{~mm} \mathrm{Hg}$. Wary of their conversion to multifocal type, lignocaine infusion was started at a rate of $2 \mathrm{mg} / \mathrm{min}$, and it was increased to $3 \mathrm{mg} / \mathrm{min}$ after 30 minutes, because of the persistence of PVCs. Lignocaine infusion was continued for150 minutes without any benefit. Repeat ABG analysis showed normal gases and potassium. During the entire period, patient's BP remained stable. No sooner did the surgeon remove the tumor, the PVCs vanished, not to return again. Lignocaine infusion was discontinued, and the rest of the surgical period was uneventful. Fentanyl and rocuronium infusions were terminated at muscle closure. Rocuronium was antagonized with sugammadex, and trachea extubated when patient was awake, with satisfactory spontaneous respiration. Estimated blood loss during surgery was $850 \mathrm{~mL}$ and urine output was $1500 \mathrm{~mL}$. She received $6.5 \mathrm{~L}$ crystalloids and half liter of colloid over a period of 5 hours. She was transferred to neurocritical care unit where she remained stable with no arrhythmias on monitor. Her postoperative ECG showed no abnormality, and her course in the hospital was uneventful.

\section{Discussion}

Various brain pathologies can lead to a range of transient and benign ECG changes which, in turn, can lead to myocardial injury and even death. ${ }^{4}$ Cardiac rhythm is modulated by ANS, which alters its function by the reflex activation of cardiac autonomic nerves in response to central autonomic commands, including those associated with stress. ${ }^{5}$ It has also 
been established that frontal cortex and heart are reciprocally connected. ${ }^{5}$ There are wide array of cortical connections between frontal cortex and IC and between prefrontal cortex (PFC) and hypothalamus. ${ }^{6}$ PFC itself modulates ANS function during stressful circumstances. ${ }^{7}$ The MPFC also indirectly regulates the ANS through projections to preautonomic nuclei in brainstem, including the nucleus of the solitary tract. ${ }^{8}$ Effects of $\mathrm{mPFC}$ on heart regulation have recently become more recognized. ${ }^{9}$ Left IC, which controls parasympathetic activity, appears to be involved in heart rate regulation, and damage to it may encourage proarrhythmia state. ${ }^{10}$ Left insular stroke is associated with an increased risk of adverse cardiac outcome and decreased cardiac wall motion compared with stroke in other locations. ${ }^{11}$

Furthermore, hypertensive neurosurgical patients are more prone to stress from balanced anesthesia technique compared with total intravenous technique, which can result in sympathetic activation and parasympathetic withdrawal. ${ }^{12}$ This, too, contributes to the aforementioned factors to engender a situation favorable for arrhythmias. Besides, another reason for reduction in parasympathetic tone (thus relative increase in sympathetic tone) in a hypertensive patient with left FL tumor might result from compression of cortical tracts between left IC and PFC, which is caused by the tumor, either due to surgical stress or from some hitherto unknown mechanism .Tilting of autonomic balance toward a relatively higher sympathetic tone generates arrhythmias, especially in a susceptible patient who might have underlying subclinical cardiac issues from long-standing hypertension.

Usual causes of intraoperative arrhythmias which include potassium imbalance, lighter planes of anesthesia, hypoxia, and hypercarbia were not present in our patient. Moreover, sevoflurane is also not arrhythmogenic. ${ }^{13}$ There were no signs and symptoms (headache/vomiting or bradycardia at presentation to operation room) suggestive of elevated intracranial pressure; importantly, the patient was on regular dexamethasone, and she also received mannitol intraoperatively. Untreated gross hypothyroidism (TSH levels greater than $10 \mathrm{mIU} / \mathrm{L}$ ) is another known factor to predispose a patient to increased incidence of ventricular arrhythmias from increased Q-T dispersion ${ }^{14}$ It was reported by Kweon and colleagues that thyroxine replacement in hypothyroid patients significantly decreased QT dispersion, thereby reducing the incidents of ventricular arrhythmias ${ }^{15}$. Our patient was on thyroxine replacement therapy and her TSH was almost normal, and we assume this precluded hypothyroidism as a possible cause for her PVCs

We hypothesize that our patient (who was hypertensive) probably had underlying subclinical coronary issue, and the condition was aggravated by pressure of tumor from stress of volatile anesthesia or some unknown mechanism, on parasympathetic projections between left IC and MPFC, or its connections with hypothalamus. This diminished the parasympathetic tone and amplified the sympathetic tone relatively, thus producing PVCs. Tumour excision restored the parasympathetic and sympathetic balance, and this resulted in cessation of PVCs.

In conclusion, in absence of obvious coronary disease, intraoperative PVCs in a hypertensive patient with left FL tumor are benign in nature, which disappear concurrent with excision of the tumor and do not need any pharmacological intervention.

\section{Conflict of Interest}

None declared.

\section{Acknowledgment}

We are thankful to Research Centre at King Fahad Medical City.

\section{References}

1 Dash M, Bithal PK, Prabhakar H, Chouhan RS, Mohanty B. ECG changes in pediatric patients with severe head injury. J Neurosurg Anesthesiol 2003;15(3):270-273

2 Benarroch EE, Chang FL. Central autonomic disorders. J Clin Neurophysiol 1993;10(1):39-50

3 Neafsey EJ. Prefrontal cortical control of the autonomic nervous system: anatomical and physiological observations. Prog Brain Res 1990;85:147-165

4 Tahsili-Fahadan P, Geocadin RG. Heart-brain axis. Effects of neurologic injury on cardiovascular function. Circ Res 2017;120(3):559-572

5 Thayer JF, Lane RD. Claude Bernard and the heart-brain connection: further elaboration of a model of neurovisceral integration. Neurosci Biobehav Rev 2009;33(2):81-88

6 Ghaziri J, Tucholka A, Girard G, et al. The corticocortical structure connectivity of the human insula. Cereb Cortex 2017;27(2):1216-1228

7 Buchanan TW, Driscoll D, Mowrer SM, et al. Medial prefrontal cortex damage affects physiological and psychological stress responses differently in men and women. Psychoneuroendocrinology 2010;35(1):56-66

8 Gabbott PL, Warner TA, Jays PRL, Salway P, Busby SJ. Prefrontal cortex in the rat: projections to subcortical autonomic, motor, and limbic centers. J Comp Neurol 2005;492(2):145-177

9 Resstel LBM, Corrêa FM. Involvement of the medial prefrontal cortex in central cardiovascular modulation in the rat. Auton Neurosci 2006;126-127:130-138

10 Oppenheimer SM, Kedem G, Martin WM. Left-insular cortex lesions perturb cardiac autonomic tone in humans. Clin Auton Res 1996;6(3):131-140

11 Laowattana S, Zeger SL, Lima JAC, Goodman SN, Wittstein IS, Oppenheimer SM. Left insular stroke is associated with adverse cardiac outcome. Neurology 2006;66(4):477-483

12 Chen Y, Jiang S, Wu Y. Effect of 2 different anesthesia methods on stress response in neurosurgical patients with hypertension or normal: a prospective clinical trial. Medicine (Baltimore 2016;95(35):e4769

13 Staikou C, Stamelos M, Stavroulakis E. Impact of anaesthetic drugs and adjuvants on ECG markers of torsadogenicity. Br J Anaesth 2014;112(2):217-230

14 Kannan L, Kotus-Bart J, Amanullah A. Prevalence of cardiac arrhythmias in hypothyroid and euthyroid patients. Horm Metab Res 2017;49(6):430-433

15 Kweon KH, Park BH, Cho CG. The effects of L-thyroxine treatment on QT dispersion in primary hypothyroidism. J Korean Med Sci 2007;22(1):114-116 